\title{
Comparison between Human Immunodeficiency Virus Positive and Negative Patients with Tuberculosis in Southern Brazil
}

\author{
Lucélia Henn ${ }^{+}$, Fabiano Nagel, Felipe Dal Pizzol
}

Serviço de Pneumologia, Hospital de Clínicas de Porto Alegre, Rua Ramiro Barcelos 2350, 2o andar, 90035-003 Porto Alegre, RS, Brasil

The objective of this study is to determine the different characteristics of human immunodeficiency virus (HIV) positive and negative patients treated for tuberculosis (TBC) in a tertiary hospital in Southern Brazil. We conducted a retrospective cohort study over a 5-year period, from January 1992 through December 1996. We reviewed medical charts of patients from our institution who received TBC treatment. We reviewed 167 medical charts of patients with confirmed TBC. HIV positivity was detected in 74 patients. There were statistically significant difference between HIV positive and negative patients in sex and age. HIV-infected patients showed significantly more signs of bacteremia than HIV-negative patients. Extra-pulmonary TBC was present respectively in 13 (17.6\%) and 21 (22.6\%) HIV positive and negative patients. There was a significant difference between chest radiograph presentation in HIV positive and negative patients. There were significantly lower hematocrit, hemoglobin, leukocyte and lymphocyte levels in HIV-positive compared to HIV-negative patients. Outcome was significantly different in the two groups with a death rate of $36.5 \%$ and $10.8 \%$ in HIV-positive and in HIV-negative patients. The difference between HIV positive and negative patients may have importance in the diagnosis, management and prognosis of patients with TBC.

Key words: tuberculosis - human immunodeficiency virus (HIV) infection - diagnosis - prognosis - Brazil

In 1990, eight million new cases of tuberculosis (TBC) were reported worldwide, and 95\% of them occurred in developing countries. In early 1992 the World Health Organization (WHO) estimated that over four million people were infected with both human immunodeficiency virus (HIV) and Mycobacterium tuberculosis (Wilkinson et al. 1996). Between 1980 and 1996, 94,997 patients with acquired immunodeficiency syndrome (AIDS) were notified to the Brazilian Ministry of Health (Ministério da Saúde 1997). In this period, 80,000 to 90,000 new TBC cases were diagnosed each year. Brazilian Ministry of Health estimates that almost 400,000 people were infected by HIV and $30 \%$ of these were also infected with $M$. tuberculosis (Kerr-Pontes et al. 1997).

The rising incidence of TBC is linked to the epidemic of HIV infection (Bloom \& Murray 1992). HIV infection increases the risk of developing active TBC, either from reactivation of latent infection or rapid progression of newly ac-

\footnotetext{
${ }^{+}$Corresponding author Fax: $+55-051-331.6699$ E-mail: pizzol@ez-poa.com.br Received 25 August 1998

Accepted 12 February 1999
}

quired infection and co-infection can enhance HIV replication, thereby shortening survival and potentially enhance HIV transmission (Ho 1996).

The infection by HIV also interferes with the diagnosis of TBC, especially in patients in the advanced phase of HIV infection (Kritski et al. 1998). The correct identification of TBC in HIV positive and negative patients are of vital importance to patient prognosis and to public health. An increase in surveillance should be done on patients at risk of co-infection by HIV (Kritski et al. 1998) and specific measures should be implemented to diminish treatment failure and transmission of multi-drug resistant M. tuberculosis. The difficult access to laboratory tests in some areas of Brazil leads to the necessity to have suspicion of co-infection of HIV and TBC only on a clinical basis.

The purpose of this study was to assess the differences existent between HIV-positive and negative TBC patients with regard to demographic characteristics, clinical features, radiographic appearance, blood chemistry, outcome and risk factors for treatment failures.

\section{PATIENTS AND METHODS}

We reviewed the inpatient medical records of all adult patients ( $>16$ years) with confirmed TBC treated at Hospital de Clínicas de Porto Alegre (HCPA), a 728-bed teaching hospital in southern 
Brazil, over a 5-year period from January 1992 through December 1996. HCPA is reference to HIV patients and is one of the hospitals in our region that can begin TBC treatment, although it is not TBC reference. Patients $<15$ years old and patients with empiric TBC treatment were excluded from the study.

TBC was diagnosed in these patients if they had (1) positive smears as shown by Ziehl-Neelsen staining, (2) isolation and the identification of $M$. tuberculosis in culture performed in Lewestein solid medium. M. tuberculosis was identified by phenotypic and biochemistry assays, or (3) evidence of granulomas on histopatologic examination of biopsied tissue, using the HE staining, plus a clinical and radiological picture suggestive of TBC (Kramer et al. 1990).

Demographic, clinical, radiographic and blood chemistry data were collected as part of normal clinical practice and transcribed on a standardized questionnaire, until the end of the treatment in the ambulatory of HCPA or in the Brazilian Public Health Service. All chest roentgenograms were categorized into three subtypes: presence of cavitation, localized disease or diffuse disease. The HIV seropositivity was confirmed by at least two positive Elisa tests associated to known risk behaviors, or by one positive Elisa test followed by a positive indirect imunofluorescence test as confirmation.

Patients were seen as an outpatient in the ambulatory of HCPA or in the Brazilian Public Health Service in the normal routine of this two services until discharge by cure or death.

Statistical analysis was performed using the Epi-Info ${ }^{\circledR}$ statistical software package. Significance tests were obtained by Yates corrected methods for categorical data. Kruskal-Wallis non-parametric test was used for continuous variables. This study was approved by the Ethical Committee of HCPA.

\section{RESULTS}

We reviewed a total of 167 confirmed TBC cases. Of this, 74 (44.3\%) were co-infected with HIV. The demographic features are demonstrated in Table I.

In HIV positive patients the most common clinical findings were fever (74\%), cough (50.7\%), weight loss $(41.1 \%)$ and clinical signals of bacteremia (chills, temperature spikes) (32.4\%) while in HIV negative the most common findings were cough $(63.4 \%)$, fever $(58.1 \%)$, weight loss $(43 \%)$ and expectoration (41.9\%) as shown in Table I.

Site of TBC was significantly different $(p<0.001)$ in HIV positive and negative patients (Table II). The most common extra-pulmonary sites of TBC in HIV positive patients were lymph nodes $(38.3 \%)$, intestine $(19.1 \%)$ and miliar pattern (14.9\%). The distribution was different in HIV negative patients, with $44.7 \%$ of cases in pleura, $13.2 \%$ miliar and $10.5 \%$ intestinal.

Radiographic presentations were demonstrated in Table II. Cavitation were more prevalent in HIV negative patients and diffuse disease in HIV posi-

TABLE I

Demographic and clinical findings in human immunodeficiency virus (HIV) positive and negative patients

\begin{tabular}{|c|c|c|c|c|}
\hline & HIV positive (\%) & HIV negative (\%) & $\mathrm{p}$ value & odds ratio \\
\hline \multicolumn{5}{|l|}{ Demographic data } \\
\hline Sex M & $59(79.7)$ & $58(62.9)$ & $<0.05$ & $0.45(0.20-0.97)$ \\
\hline $\mathrm{F}$ & $11(20.3)$ & $31(37.1)$ & & \\
\hline \multicolumn{5}{|l|}{ Ethnic group } \\
\hline Black & $17(24.3)$ & $14(15.7)$ & NS & $0.58(0.24-1.38)$ \\
\hline White & $53(75.7)$ & $75(84.3)$ & & \\
\hline Age (mean) & 33.4 & 46.1 & $<0.001$ & \\
\hline \multicolumn{5}{|l|}{ Clinical findings } \\
\hline Fever & $54(74)$ & $54(58.1)$ & 0.04 & $2.05(1.00-4.25)$ \\
\hline Cough & $37(50.7)$ & $59(63.4)$ & NS & $0.59(0.30-1.17)$ \\
\hline Weight loss & $30(41.1)$ & $40(43)$ & NS & $0.92(0.47-1.81)$ \\
\hline Bacteremia & $23(32.4)$ & $5(5.5)$ & $<0.001$ & $8.10(2.67-26.31)$ \\
\hline Fatigue & $21(28.8)$ & $15(16.1)$ & 0.04 & $2.10(0.93-4.79)$ \\
\hline Expectoration & $21(28.8)$ & $39(41.9)$ & NS & $0.56(0.27-1.14)$ \\
\hline Dyspnea & $18(24.7)$ & $33(35.5)$ & NS & $0.60(0.28-1.25)$ \\
\hline Night sweats & $12(16.4)$ & $17(18.3)$ & NS & $0.88(0.36-2.14)$ \\
\hline Hemoptysis & $4(5.5)$ & $5(5.5)$ & NS & $1.02(0.22-4.65)$ \\
\hline
\end{tabular}

NS: non-significant differences. 
TABLE II

Tuberculosis site, radiographic and biochemical findings in human immunodeficiency virus (HIV) positive and negative patients

\begin{tabular}{lccc}
\hline Tuberculosis site & HIV positive $(\%)$ & HIV negative $(\%)$ & p value (chi-squares) \\
\hline Pulmonary & $27(36.5)$ & $55(59.1)$ & $0.001(15.14)$ \\
Extra-pulmonary & $13(17.6 \%)$ & $21(22.6)$ & \\
Both & $34(45.9)$ & $117(18.3)$ & \\
Chest radiographs & & & \\
Cavitation & $5(8.2)$ & $27(40.3)$ & $0.001(13.15)$ \\
Localized disease & $19(31.1)$ & $33(49.3)$ & \\
Diffuse disease & $35(57.4)$ & $7(10.4)$ & $0.001(13.05)$ \\
No alterations & $2(3.3)$ & $0(0)$ & \\
Presence of lymph nodes & $8(12.9)$ & $1(1.2)$ & $0.001(39.98)$ \\
Biochemical findings (means) & & & $0.001(36.35)$ \\
Hematocrit & $29.3 \%$ & $36 \%$ & $0.01(14.67)$ \\
Hemoglobin & $9.25 \mathrm{mg} / \mathrm{dl}$ & $11.33 \mathrm{mg} / \mathrm{dl}$ & $0.01(12.87)$ \\
Leukocyte & $6692 \mathrm{cells} / \mathrm{ml}$ & $9184 \mathrm{cells} / \mathrm{ml}$ & \\
Lymphocyte & $1072 \mathrm{cells} / \mathrm{ml}$ & $1677 \mathrm{cells} / \mathrm{ml}$ & \\
\hline
\end{tabular}

tive patients. Hilar or mediastinal lymph nodes were more common in HIV positive patients $(p<0.005)$. Biochemistry measurements differed in the two groups ( $\mathrm{p}<0.01$ in all values) as demonstrated in Table II.

The major diagnostic method in both HIV positive and negative patients were positive smears as shown by Ziehl-Neelsen staining (71\% against $76 \%$, respectively). The isolation and identification of M. tuberculosis in culture were positive in $32 \%$ of HIV patients and in $27 \%$ of HIV negative patients. The evidence of micobacterial infection on histopatologic examination of biopsied tissue was responsible for $34 \%$ of diagnosis in HIV positive and for $35 \%$ in HIV negative patients.

Clinical outcomes were significantly different between the two groups $(\mathrm{p}<0.001)$ as shown in Table III. We verified a high death rate in HIV positive patients $(36.5 \%)$ in comparison with HIV negative patients $(10.8 \%)$. Treatment was abandoned by $9(12.2 \%)$ HIV positive patients and by $4(4.3 \%)$ HIV negative patients. The vast majority of both HIV positive and negative patients were treated with first line agents (isoniazid+rifampin+ pyrazinamide) according to the standard protocol

TABLE III

Outcomes in human immunodeficiency virus (HIV) positive and negative patients

\begin{tabular}{lccc}
\hline Outcome & $\begin{array}{c}\text { HIV } \\
\text { positive }(\%)\end{array}$ & $\begin{array}{c}\text { HIV } \\
\text { negative }(\%)\end{array}$ & $\begin{array}{c}\text { p value } \\
\text { (chi-squares) }\end{array}$ \\
\hline Death & $27(36.5)$ & $10(10.8)$ & $0.01(11.35)$ \\
Cure & $33(44.5)$ & $78(83.8)$ & \\
Recurrence & $5(6.8)$ & $1(1.1)$ & \\
Abandoned & $9(12.2)$ & $4(4.3)$ & \\
\hline
\end{tabular}

of Brazilian Ministry of Health.

In HIV negative patients only signs of bacteremia were associated to higher death rates; $80 \%$ of the patients with this signs died during treatment, while $6.8 \%$ of patients, without bacteremia signs died $(\mathrm{p}<0.001)$. In contrast, HIV positive patients had more variables associated with higher death rates: signs of bacteremia, hematocrit levels, leukocyte and lymphocyte counts. Only $15.2 \%$ of HIV positive patients with signs of bacteremia survived in contrast with and survival rate of $56 \%$ in patients without these signs $(\mathrm{p}<0.01)$. The mean hematocrit was 27.6 and 30.1 respectively in patients who died and survived $(\mathrm{p}<0.05)$. The mean leukocyte count was 4950 cells $/ \mathrm{ml}$ and mean lymphocyte count was 666 cells $/ \mathrm{ml}$ in patients who died in comparison with leukocyte counts of 7468 cells $/ \mathrm{ml}$ and lymphocyte counts of 1336 cells $/ \mathrm{ml}$ in surviving patients $(\mathrm{p}<0.01)$.

\section{DISCUSSION}

The rising incidence in seroprevalence of HIV infection among TBC patients leads to health professionals to become more aware of the different presentations of TBC in HIV positive patients. It is of vital importance the suspicion of HIV infection in a patient with TBC, principally patients with atypical TBC presentations. This study shows that several clinical, laboratorial and radiographic features occur in different proportions in patients infected by both HIV and TBC compared with TBC patients not infected with HIV.

In our study, HIV positive TBC patients were younger and predominantly male. Handwerger et al. (1991) and Batungwanayo et al. (1992) also demonstrated that HIV positive TBC patients tend 
to be younger and predominantly male compared to HIV negative TBC patients.

The clinical picture was different in HIV positive and negative patients, but only fever, fatigue and clinical signs of bacteremia were significantly more prevalent in HIV positive patients. Batungwanayo et al. (1992) found that fever was the only clinical symptom more prevalent in HIV patients with TBC when compared with those HIV negative. Shafer et al. (1989) demonstrated that $M$. tuberculosis bacteremia occurs more frequently in HIV positive patients.

In our study, the majority of HIV negative patients presented pulmonary disease in contrast to HIV positive patients, that predominantly showed disseminated disease. Sunderam et al. (1986) and Given et al. (1994) have shown the higher percentage of both pulmonary and extra-pulmonary disease in HIV positive patients.

The radiographic patterns were also different in the two groups. HIV positive patients characteristically presented diffuse infiltrates in contrast with localized or cavitary disease in HIV negative patients. Saks and Posner (1992) also demonstrated that the positivity in HIV status was linked to a significantly higher percentage of lymphadenopathy and diffuse infiltrates in chest roentgenograms when compared with a seronegative group. Pitchenik and Rubinson (1985) found a significantly higher prevalence of adenopathy, upper and lower localized lung field infiltrates, and a very significant lower prevalence of pulmonary cavities in patients with TBC and HIV infection compared with those without it. Pozniak et al. (1995) failed to show any characteristic pattern differentiating HIV positive and negative patients, except for the predominance of cavitation in HIV negative patients. Therefore, diffuse pattern in a chest film in a patient with known pulmonary TBC should alert the physician to the possibility of concurrent HIV infection. On the other hand, the diffuse presentation of pulmonary TBC in an HIV positive patient would probably difficult the differential diagnosis with other opportunistic infections.

Importantly, HIV positive TBC patients had poorer outcome (death and treatment abandoning) than HIV negative patients in our study. Perriëns et al. (1991) demonstrated that the mortality during the year after the begining of TBC treatment among HIV patients is significantly higher (over 30\% absolute mortality difference between groups) than in HIV negative ones. Similarly, Stoneburner et al. (1992) showed a mortality rate 7.5 times higher in HIV positive than in HIV negative patients.

One possible bias in our study was the fact that the confirmation of TBC diagnosis includes culture as well as positive smears and histologic find- ings. It is quite possible that some of these patients with positive smears or suggestive histologic findings had non tuberculous mycobacteria rather than TBC. It is important to note that the culture of atypical mycobacteriosis is not made routinely in the vast majority of Brazilian hospitals. In addition, the prevalence of atypical mycobacteriosis in our region is not a major problem as it was in more developed countries. In January 1997, our institution introduced the isolation of atypical mycobacteriosis and in a survey we found only 17 cases of atypical mycobacteriosis since the introduction of this new test (Henn et al. 1998). Our results demonstrated the reality in a tertiary hospital which, theoretically, serve more severe patients or patients in whom the diagnosis is more difficult. So, this can difficult the extrapolation of the results principally to ambulatory patients. The reality of our Public Health Service, where patients goes direct for the tertiary hospital even before looking for medical care in the primary level, can diminish this bias and make our results more close to the TBC reality in our region.

The clinical, radiographic and prognostic differences between HIV positive patients with TBC and those with TBC but without HIV infection are of paramount importance when considering areas of relatively high TBC prevalence, and should be taken into account whenever considering a diagnosis of HIV infection and its potential for TBC co-infection, and vice-versa.

Our work suggest that health professionals can have some accessible clues to suspect of co-infection with TBC and HIV. This can be of special interest once respected organizations like World Health Organization and Centers for Diseases Control and Prevention recommended the precocious identification of HIV infection in a TBC patient as one of the priorities in the management of TBC (Kritski et al. 1995). When confirmed the diagnosis of TBC and HIV infection the surveillance must be more close once therapeutic failure (i.e. noncompliance, adverse drug reactions and death) are more prevalent in this group.

\section{REFERENCES}

Batungwanayo J, Taelman H, Dhote R, Bogaerts J, Allen S, Van de Perre P 1992. Pulmonary tuberculosis in Kigali, Rwanda. Am Rev Respir Dis 146: 53-56.

Bloom BR, Murray CJ 1992. Tuberculosis: commentary on a re-emergent killer. Science 257: 1055-1064.

Given MJ, Khan A, Reichman LB 1994. Tuberculosis among patients with AIDS and a control group in an inner-city community. Arch Int Med 154: 640-645.

Handwerger S, Mildvan D, Senie R, McKinley FW 1987. Tuberculosis and the acquired immunodeficiency syndrome at a New York city hospital: 1978-1985. Chest 100: 176-180. 
Henn L, Wolfart M, Dal Pizzol F 1998. Atypical mycobacteriosis in HIV positive patients in Southern Brazil. Chest 114 (Suppl): 288s.

Ho JL 1996. Co-infection with HIV and Mycobacterium tuberculosis: immunologic interactions, disease progression, and survival. Mem Inst Oswaldo Cruz 91 : 385-387.

Kerr-Pontes LRS, Oliveira FAZ, Freire CAM 1997. Tuberculosis associated with AIDS: the position in a northeastern region of Brazil. Rev Saú Púb 31: 323329.

Kramer F, Modilevski T, Waliany AR, Leedon JM, Barnes PF 1990. Delayed diagnosis of tuberculosis in patients with human immunodeficiency virus infection. Am J Med 89: 451-456.

Kritski AL, Silva JRL, Conde MB 1998.Tuberculosis and HIV: renewed challenge. Mem Inst Oswaldo Cruz 93: 417-421.

Kritski AL, Dalcolmo M, Bianco R, Melo FF, Pinto WP, Schechther M, Castelo A 1995. Associação tuberculose e infecção pelo HIV no Brasil. Bol Of Sanit Panam 118: 542-554.

Ministério da Saúde do Brasil 1997. AIDS: Boletim Epidemiológico 5: 3-33.

Perriëns JH, Colebunders RL, Karahunga C, Willame JC, Jeugmans J, Kaboto M, Mukadi Y, Pauwels P, Ryder RW, Prignot J 1991. Increased mortality and tuberculosis treatment failure rate among human immunodeficiency virus (HIV) seropositive compared with HIV seronegative patients with pulmonary tuberculosis treated with "standard" chemo- therapy in Kinshasa, Zaire. Am Rev Respir Dis 144: 750-755.

Pitchenik AE, Rubinson HA 1985. The radiographic appearance of tuberculosis in patients with the acquired immune deficiency syndrome (AIDS) and pre-AIDS. Am Revi Respir Dis 131: 393-396.

Pozniak AL, MacLeod A, Ndlovu D, Ross E, Mahari M, Weinberg J 1995. Clinical and chest radiographic features of tuberculosis associated with human immudeficiency virus in Zimbabwe. Am J Resp Crit Care Med 152: 1558-1561.

Saks AM, Posner R 1992. Tuberculosis in HIV positive patients in South Africa: a comparative radiological study with HIV negative patients. Clin Radiol 46: 387-390.

Shafer RW, Goldberg R, Sierra M, Glatt AE 1989. Frequency of Mycobacterium tuberculosis bacteremia in patients with tuberculosis in an area endemic for AIDS. Am Rev Respir Dis 140: 1611-1613.

Stoneburner R, Laroche E, Prevots R, Singh T, Blum S, Terry P, Reatrice S, Adler J 1992. Survival in a cohort of human immunodeficiency virus-infected tuberculosis patients in New York City. Arch Intern Med 152: 2033-2037.

Sunderman G, McDonald RJ, Maniatis T, Oleske J, Kapila R, Reichman LB 1986. Tuberculosis as a manifestation of the acquired imunodeficiency syndrome (AIDS). J Am Med Assoc 256: 362-366.

Wilkinson D, Moore DAJ 1996. HIV-related tuberculosis in South Africa - Clinical features and outcome. S Afr Med J 86: 64-67. 
382 HIV Positive and Negative Patients with TBC • Lucélia Henn et al. 